\title{
Jain-Kivelson-type resonance as a noninvasive probe of screening in the quantum Hall regime
}

\author{
R. Nemutudi ${ }^{\mathrm{a}}$, C.-T. Liang ${ }^{\mathrm{a}, \mathrm{b}, *}$, M.J. Murphy ${ }^{\mathrm{a}}$, H.E. Beere ${ }^{\mathrm{a}}$, C.G. Smith ${ }^{\mathrm{a}}$, D.A. Ritchie ${ }^{\mathrm{a}}$, \\ M. Pepper ${ }^{\mathrm{a}}$, G.A.C. Jones ${ }^{\mathrm{a}}$

 \\ ${ }^{\mathrm{b}}$ Department of Physics, National Taiwan University, Taipei 106, Taiwan, ROC
}

Available online 9 April 2005

\begin{abstract}
It has been shown that the local conditions in an electrical circuit can affect a nearby, but electrically isolated circuit. Such a 'noninvasive' measurement technique can be used to measure the screening ability of an electron system. In this work, we use a one-dimensional (1D) channel to screen an in-plane electric field between a gate and another 1D channel. We observe resistance resonances arising from tunnelling between counter-propagating edge channels via a localised state which is formed due to the presence of the disorder potential within our 1D channels. Using the observed resistance resonance as a noninvasive probe, we are able to investigate the screening ability (compressibility) of a 1D channel in the quantum Hall regime. Our results suggest that $\sim 73 \%$ of the electric field can penetrate a 1D channel in the quantum Hall regime. (C) 2005 Elsevier Ltd. All rights reserved.
\end{abstract}

PACS: 73.40.Gk; 73.20.Dx

Keywords: Noninvasive; Jain-Kivelson-type; Resonance

\section{Introduction}

It has been shown that a GaAs two-dimensional electron gas (2DEG) can only partially screen an external electric field from an electrode [1]. In the seminal work of Luryi [2], it has been suggested that partial penetration of an electric field through a highly conductive 2DEG allows the implementation of novel high-speed devices, such as a three-terminal resonant tunnelling transistor and a gatedcontrolled thermionic emission transistor.

Resonant tunnelling through a single energy level has become a subject of intensive theoretical and experimental interest since the advent of quantum mechanics. It is known that in a narrow quantum Hall-effect device, a resonance can be observed due to tunnelling between extended states via a localised state [3]. Such a resonance could manifest itself as a resistance peak in the integer quantum Hall regime.

\footnotetext{
* Corresponding author. Address: Department of Physics, National Taiwan University, Taipei 106, Taiwan, ROC. Fax: +886223639984.

E-mail address: ctliang@phys.ntu.edu.tw (C.-T. Liang).
}

0026-2692/\$ - see front matter (C) 2005 Elsevier Ltd. All rights reserved. doi:10.1016/j.mejo.2005.02.104
'Noninvasive' measurement technique utilises the fact that the local conditions in an electrical circuit can affect a nearby, but electrically isolated circuit [4]. Such measurement technique can be used to probe the screening characteristics of an electron system [5]. In this work, we use a one-dimensional (1D) channel to screen an in-plane electric field between a gate and another 1D channel. Due to the presence of the disorder potential within our 1D channels, we observe resistance resonances arising from tunnelling between counter-propagating edge states via a localised state. We are able to investigate the screening ability (compressibility) of a 1D channel in the quantum Hall regime using the observed resistance resonance as a noninvasive probe. Using a simple model, we estimate that $\sim 73 \%$ of the electric field can penetrate a 1D channel in a Landau level filling factor $\nu=1$ quantum Hall state.

\section{Experimental}

The device we studied comprises of two closely spaced, but electrically isolated 1D channels fabricated using local anodic oxidation induced by atomic force microscope (AFM) [6-9]. The inset to Fig. 1 shows an AFM image of 


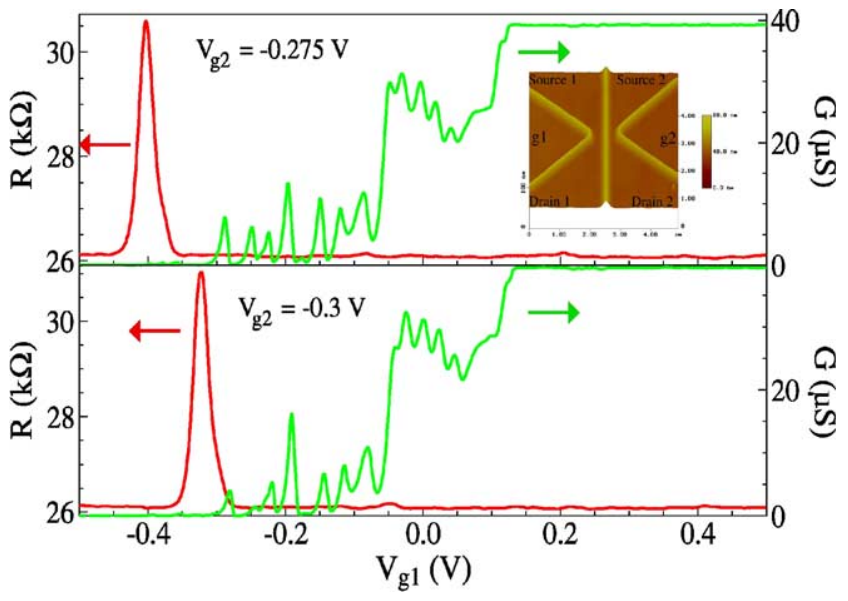

Fig. 1. Conductance $(G)$ measurements of channel 1 and resistance $(R)$ measurement of channel 2 as a function of the voltage $V_{\mathrm{g} 1}$ applied on $(\mathrm{g} 1)$ at $V_{\mathrm{g} 2}=-0.275 \mathrm{~V}$ and $B=7 \mathrm{~T}$. (b) $G\left(V_{\mathrm{g} 1}\right)$ and $R\left(V_{\mathrm{g} 1}\right)$ at $V_{\mathrm{g} 2}=-0.3 \mathrm{~V}$ and $B=7 \mathrm{~T}$. The inset shows an AFM image of a typical device. The PL spectra of $\operatorname{InAs}(\mathrm{N}) / \mathrm{InGaAs} \mathrm{SQW}$ taken at temperature $10 \mathrm{~K}$.

a typical device. The carrier concentration of the $2 \mathrm{DEG}$ was $4.32 \times 10^{15} \mathrm{~m}^{-2}$ with a mobility of $53.7 \mathrm{~m}^{2} / \mathrm{V} \mathrm{s}$ in the dark. The depth of the $2 \mathrm{DEG}$ is $36 \mathrm{~nm}$. Experiments were performed in a dilution refrigerator at a base temperature $T \sim 50 \mathrm{mK}$. Two-terminal conductance of channel 1 and two-terminal resistance of channel 2 were measured simultaneously using standard phase-sensitive techniques.

Fig. 1(a) shows conductance $(G)$ of channel 1 and resistance $(R)$ of channel 2 as a function of the voltage $V_{\mathrm{g} 1}$ applied on $\mathrm{g} 1$ at $V_{\mathrm{g} 2}=-0.275 \mathrm{~V}$ and $B=7 \mathrm{~T}$. The conductance and resistance plateaus correspond to a $\nu=1$ spin-split integer quantum Hall state. The conductance resonances can be ascribed to Coulomb oscillations in the quantum Hall regime [10]. These oscillations are possibly due to tunnelling through a quantum-dot-like structure as a result of the disorder potential $[11,12]$ within channel 1 . We ascribe the resistance peak to a resonance which results from tunneling between counter-propagating $\nu=1$ edge channels via a localised state.

Fig. 1(b) shows $G\left(V_{\mathrm{g} 1}\right)$ and $R\left(V_{\mathrm{g} 1}\right)$ at $V_{\mathrm{g} 2}=-0.3 \mathrm{~V}$ and $B=7 \mathrm{~T}$. Compared with the data shown in Fig. 1(a), in order to observe the same resistance resonance, it is evident that a less negative voltage in $V_{\mathrm{g} 1}$ is required as shown in Fig. 1(b). By increasing $V_{\mathrm{g} 2}$ in steps, we can investigate the evolution of the same resistance resonant structure in the $V_{\mathrm{g} 1}-V_{\mathrm{g} 2}$ plane. Such results are shown in Fig. 2 as a grey-scale plot of $G\left(V_{\mathrm{g} 1}\right)$ at different values of $V_{\mathrm{g} 2}$. We have subtracted the variation of the background in $G\left(V_{\mathrm{g} 1}\right)$ in order to increase the contrast. Black (white) regions correspond to conductance maxima (minima) in $G\left(V_{\mathrm{g} 1}\right)$. The grey areas correspond to plateau-like structures. The white symbols correspond to the positions of the resistance peaks in the $V_{\mathrm{g} 1}-V_{\mathrm{g} 2}$ plane. As shown in Fig. 2, there is a good linear fit for $V_{\mathrm{g} 1}<0$. For $V_{\mathrm{g} 1}>0.1 \mathrm{~V}$, there is also a good linear fit with a less negative slope compared with that for $V_{\mathrm{g} 1}<0$.

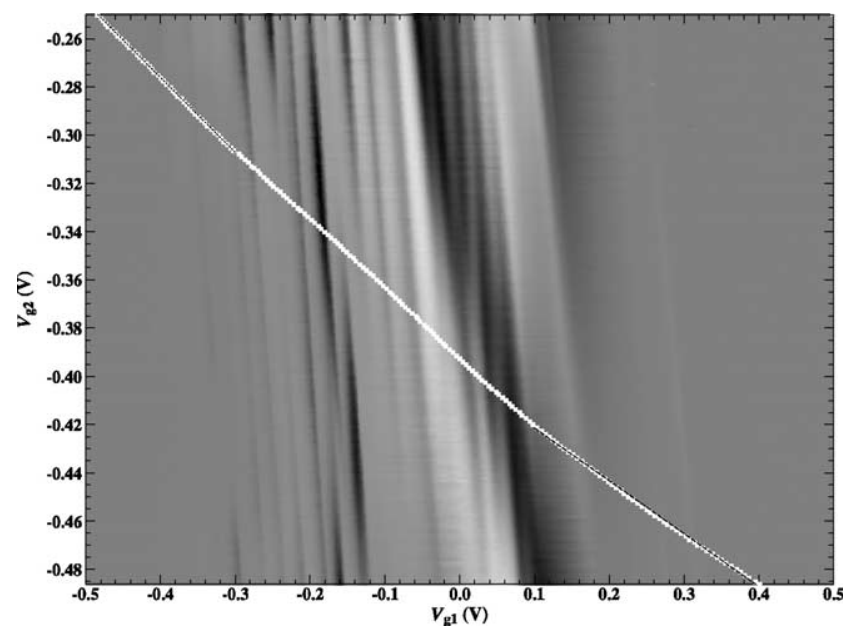

Fig. 2. A grey-scale plot of $G\left(V_{\mathrm{g} 1}\right)$ at $B=7 \mathrm{~T}$ at different $V_{\mathrm{g} 2}$. The white symbols correspond to the positions of the observed resistance resonances in the $V_{\mathrm{g} 1}-V_{\mathrm{g} 2}$ plane. There are two good linear fits for $V_{\mathrm{g} 1}<0 \mathrm{~V}$ (shown in the dotted line) and $V_{\mathrm{g} 1}>0.1 \mathrm{~V}$ (shown in the dashed line), respectively.

We observe a cross over region $0 \leq V_{\mathrm{g} 1} \leq 0.1 \mathrm{~V}$, between these two fits.

For a fixed distance, the electric fields emanating from the in-plane gates $\mathrm{g} 1$ and $\mathrm{g} 2$ are approximately proportional to the applied gate voltages. This is supported by the fact that the observed Coulomb blockade oscillations in a lateral quantum dot fabricated using the same local anodic oxidation fabrication technique are periodic in gate voltage [10]. The slope $\mathrm{d} V_{\mathrm{g} 2} / \mathrm{d} V_{\mathrm{g} 1}$ in the $V_{\mathrm{g} 1}-V_{\mathrm{g} 2}$ plane reflects the ratio $C_{12} / C_{22}$, where $C_{22}$ and $C_{12}$, respectively, denote the capacitance of $\mathrm{g} 2$ to channel 2 and the capacitance of $\mathrm{g} 1$ to channel 2 through the intervening 1D-channel. The slope of the linear fit through our experimental data (shown in white symbols) in the $V_{\mathrm{g} 1}-V_{\mathrm{g} 2}$ plane can therefore be regarded as a noninvasive probe of the screening characteristics of the intervening 1D-channel.

For $V_{\mathrm{g} 1} \lesssim-0.3 \mathrm{~V}$, the conductance through channel 1 is almost zero. In this case, the electrons within channel 1 are depleted and thus no screening is experienced by $\mathrm{g} 1$. Under such conditions, the evolution of the resistance resonance in the $V_{\mathrm{g} 1}-V_{\mathrm{g} 2}$ plane is the ratio $C_{12} / C_{22}$ of the capacitive coupling of $\mathrm{g} 2$ and $\mathrm{g} 1$ to channel 2. If $V_{\mathrm{g} 2}$ is made less negative, a smaller electric field is emitted from $\mathrm{g} 2$, and therefore a more negative voltage on $\mathrm{g} 1$ (larger electric field from g1) is required to bring the localised state into a resonance. One could thus consider the electric field experienced by the localised state in channel 2 as being approximately constant. From the linear fit for $V_{\mathrm{g} 1}<0$, we know $\mathrm{d} V_{\mathrm{g} 2} / \mathrm{d} V_{\mathrm{g} 1}$ is -0.291 . As $\mathrm{g} 2$ is closer to channel 2 than is $\mathrm{g} 1$, one would thus expect $\mathrm{d} V_{\mathrm{g} 2} / \mathrm{d} V_{\mathrm{g} 1}$ to be less than unity, consistent with our results. It is interesting to note that the linear fit for $V_{\mathrm{g} 1}<-0.3 \mathrm{~V}$ extends deep into the $\nu=0$ to $\nu=$ 1 transition regions. This could be due to the fact that electrons travel through a disordered potential landscape in a complicated manner in this regime which is evidenced by the observed resonant features in $G\left(V_{\mathrm{g} 1}\right)$. 
Therefore the expected enhancement in screening from the bulk state may not occur. For $V_{\mathrm{g} 1}>0.1 \mathrm{~V}$, our results show that $\mathrm{d} V_{\mathrm{g} 2} / \mathrm{d} V_{\mathrm{g} 1}$ is -0.21 .

We now consider the implications of our results. For $V_{\mathrm{g} 1}<0$, if $\mathrm{d} V_{\mathrm{g} 2}$ is decreased by $0.01 \mathrm{~V}$, in order to observe the same resistance resonance, $\mathrm{d} V_{\mathrm{g} 1}$ has to be increased by $0.035 \mathrm{~V}$. The situation is different for the $\nu=1$ regions. If $\mathrm{d} V_{\mathrm{g} 2}$ is decreased by the same amount $(0.01 \mathrm{~V})$, then $\mathrm{d} V_{\mathrm{g} 1}$ has to be increased by $0.0476 \mathrm{~V}$ in order to observe the same peak. For a fixed $V_{\mathrm{g} 2}=0.01 \mathrm{~V}$, such an increase in $\mathrm{d} V_{\mathrm{g} 1}$ $(0.0476 \mathrm{~V}$, which is considerably larger than $0.035 \mathrm{~V})$ can be ascribed to the finite screening ability of channel 1 in the $\nu=1$ quantum Hall state. As stated earlier, the electric field emanating from $\mathrm{g} 1$ is proportional to the applied gate voltage $V_{\mathrm{g} 1}$. Our results therefore suggest that a fraction of $0.035 / 0.0476 \sim 73 \%$ of the electric field from g1 can penetrate channel 1 at $\nu=1$ if we neglect any parasitic capacitance in our system.

In conclusion, we have studied two closely spaced, but electrically isolated 1D channels fabricated using local anodic oxidation induced by AFM. In the quantum Hall regime, we observe resistance resonances arising from tunnelling between two extended states via a localised state formed due to an impurity potential. Using the evolution of the resistance resonance as a noninvasive probe, we can demonstrate the screening ability of a 1D channel in the quantum Hall regime.

\section{Acknowledgements}

This work was funded by the EPSRC, United Kingdom, the NSC, Taiwan (42006F) and the NRF, South Africa.

\section{References}

[1] J.P. Eisenstein, L.N. Pfeiffer, K.W. West, Phys. Rev. B 50 (1994) 1760.

[2] S. Luryi, Appl. Phys. Lett. 52 (1987) 501.

[3] J.K. Jain, S.A. Kivelson, Phys. Rev. Lett. 60 (1988) 1542.

[4] M. Field, C.G. Smith, M. Pepper, D.A. Ritchie, J.E.F. Frost, G.A.C. Jones, D.G. Hasko, Phys. Rev. Lett. 70 (1993) 1311.

[5] M. Field, C.G. Smith, M. Pepper, K.M. Brown, E.H. Linfield, M.P. Grimshaw, D.A. Ritchie, G.A.C. Jones, Phys. Rev. Lett. 77 (1996) 350.

[6] R. Held, T. Heinzel, P. Studerus, K. Ensslin, M. Holland, Appl. Phys. Lett. 71 (1997) 2689.

[7] U.F. Keyser, H.W. Schumacher, U. Zeitler, R.J. Haug, K. Eberl, Appl. Phys. Lett. 76 (2000) 457.

[8] R. Nemutudi, N.J. Curson, N.J. Appleyard, D.A. Ritchie, G.A.C. Jones, Microelectron. Eng. 57 (2001) 967.

[9] R. Nemutudi, M. Kataoka, C.J.B. Ford, N.J. Appleyard, M. Pepper, D.A. Ritchie, G.A.C. Jones, J. Appl. Phys. 95 (2004) 2557.

[10] D.H. Cobden, C.H.W. Barnes, C.J.B. Ford, Phys. Rev. Lett. 82 (1999) 4695.

[11] J.T. Nicholls, J.E.F. Frost, M. Pepper, D.A. Ritchie, M.P. Grimshaw, G.A.C. Jones, Phys. Rev. B 48 (1994) 8866.

[12] C.-T. Liang, I.M. Castleton, J.E.F. Frost, C.H.W. Barnes, C.G. Smith, C.J.B. Ford, D.A. Ritchie, M. Pepper, Phys. Rev. B 55 (1997) 6723. 